\title{
ANÁLISE DO FLUXO DE DISTRIBUIÇÃO E ESTORNO DE MEDICAMENTOS EM HOSPITAL FILANTRÓPICO DE SANTA MARIA, RS ${ }^{1}$
}

\author{
ANALYSIS OF DISTRIBUITION FLOW AND CANCELLATION IN A \\ PHILANTHROPIC HOSPITAL OF SANTA MARIA, RS
}

\section{ANÁLISIS DE LA INVERSIÓN DE FLUJO Y DISTRIBUCIÓN DE DROGAS EN HOSPITAL FILANTRÓPICO DE SANTA MARIA, RS}

\author{
Jane Beatriz Limberger \\ Centro Universitário Franciscano \\ janebeatriz@unifra.br
}

Daniel de Azevedo Ferrony

Secretaria Municipal de Saúde de São Grabriel

daniel.ferrony@hotmail.com

\author{
Tatieli Sampaio dos Santos \\ Centro Universitário Franciscano \\ tati.ssantos@hotmail.com
}

\author{
Silvana Maria Michelin Bertagnolli \\ Centro Universitário Franciscano \\ silvibert@yahoo.com.br
}

\author{
Karoline de Campos Prediger \\ Universidade Federal do Paraná \\ karol_obs@hotmail.com
}

\begin{abstract}
RESUMO
A gestão de medicamentos na farmácia hospitalar demanda um controle rígido de processos a fim de otimizar o fluxo de produtos para as unidades bem como seu retorno à farmácia quando não utilizados. Este trabalho objetivou realizar a conferência do estorno dos medicamentos para a farmácia de hospital filantrópico, analisando o sistema de medicamentos e identificando as causas e padrões de erros de devolução. Foi realizada uma análise descritiva longitudinal por meio de prescrições médicas, registrando os erros contidos nas devoluções dos medicamentos e materiais médicos. Foram verificadas prescrições de quatro unidades do hospital, sendo que a unidade psiquiátrica apresentou menor porcentagem de erros na devolução de medicamentos. Visualizou-se grande quantidade de erros referente as unidades clínica adulta e pós-cirúrgica $(47,49 \%$ e 54,35\%, respectivamente). Com o objetivo de reduzir erros de medicação e estorno, faz-se necessária a adoção de procedimentos operacionais padrão, dose unitária, promoção e capacitação permanente e a educação farmacêutica, além da incorporação da prescrição eletrônica e código de barras no processo de administração de medicamentos.
\end{abstract}

Palavras-chave: farmácia hospitalar, erros de estorno, gestão farmacêutica.

\begin{abstract}
The management of medicines in the hospital pharmacy demand tight control of processes in order to optimize the flow of products to the units as well as his return to the pharmacy when not in use. This study aimed to make the conference the reversal of medicines to the pharmacy of philanthropic hospital, analyzing the system of medicines and identifying the causes and patterns of errors return. We performed a longitudinal descriptive analysis through prescriptions, logging the errors contained in the returns of medicines and medical supplies. Were observed prescriptions of four units of the hospital, and the psychiatric unit had lower percentage of errors in the return of drugs. Was visualized lot of errors regarding adult clinical units and postoperative $(47.49 \%$ and $54.35 \%$, respectively). Aiming to reduce medication errors and cancelation, it is necessary to adopt standard operating procedures, unit dose, promotion and training and continuing pharmacy education, besides the incorporation of electronic prescription and bar code on the medication administration process.
\end{abstract}

Keywords: hospital pharmacy, reversal errors, pharmaceutical management.

\section{RESUMEN}

\footnotetext{
${ }^{1}$ Submetido em 17 de Janeiro de 2013. Aceito em 04 de Abril de 2013. O artigo foi avaliado segundo o processo de duplo anonimato além de ser avaliado pelo editor. Editores responsáveis: Márcio Augusto Gonçalves e Lucas Maia dos Santos. Reprodução parcial ou total e trabalhos derivativos permitidos com a citação apropriada da fonte.
} 
La gestión de los medicamentos en la farmacia del hospital requiere un proceso de control estricto con el fin de optimizar el flujo de productos a las unidades, así como su retorno a la farmacia cuando no se utiliza. Este estudio tuvo como objetivo hacer que la rueda de la reversión de los medicamentos a la farmacia del hospital filantrópico, el análisis del sistema de drogas y la identificación de las causas y los patrones de errores regreso. Se realizó un análisis descriptivo de longitudinal a través de recetas, el registro de los errores contenidos en las declaraciones de los medicamentos y suministros médicos. Las recetas se comprobaron cuatro unidades del hospital, y la unidad psiquiátrica mostró menor porcentaje de errores en la devolución de los medicamentos. Gran cantidad visualizada de errores con respecto a las unidades de adulto clínica y post-quirúrgica $(47,49 \%$ y $54,35 \%$, respectivamente). Con el objetivo de reducir los errores de medicación y cancelación, es necesario adoptar procedimientos operativos estándar, dosis unitarias, la promoción y la formación continua y la educación farmacéutica, y la incorporación de la prescripción electrónica y código de barras en el proceso de administración de medicamentos.

Palabras clave: farmacia hospitalaria, los errores de inversión, la gestión farmacéutica.

\section{1 - INTRODUÇÃO}

A farmácia hospitalar tem por principal função responder à demanda das necessidades de medicamentos de pacientes hospitalizados, abrigando os estoques destes produtos (GONÇALVES, NOVAES E SIMONETTI, 2006). Desta forma, a organização para a dispensação dos medicamentos na área hospitalar é de grande importância, constituindo um sistema complexo, composto por vários processos que visam a administração do medicamento ao paciente de maneira ágil e segura. Segundo Miasso e colaboradores (2006) a compreensão destas atividades ocorre com a prática, identificando vários componentes necessários para realizar o propósito de fornecer tratamento medicamentoso qualificado e racional.

Em um hospital, o gerenciamento dos medicamentos e materiais médicos compõe uma cadeia de atividades para que os mesmos sejam distribuídos em vários setores, a fim de manter a qualidade do serviço prestado ao paciente (AGAPITO, 2005). O ciclo do medicamento nos hospitais é bastante complexo e composto de vários processos. Dentro do sistema de distribuição, existem vários tipos de fluxo, dentre os quais se podem citar o coletivo, individualizado, combinado ou misto e dose unitária. Ainda que o número e o tipo de processos variem de um hospital para o outro, estima-se que de 20 a 60 etapas diferentes estejam envolvidas nos processos de prescrição, dispensação e administração de medicamentos. O envolvimento de muitos profissionais dá a esse ciclo a característica multidisciplinar e gera múltiplas transferências de pedidos ou matérias, que podem favorecer a ocorrência de erros. Quanto maior o número de elementos de um sistema, menor a probabilidade de que cada elemento opere com sucesso (OLIVEIRA E MELO, 2011).

Os processos e profissionais envolvidos no sistema de distribuição de medicamento são sumarizados na Figura 1. De uma maneira geral, o medicamento sai da farmácia mediante a apresentação de uma prescrição médica, sendo o médico o responsável por esta etapa. A dispensação e distribuição dos medicamentos que passaram pela etapa anterior são de responsabilidade dos farmacêuticos, apesar de alguns técnicos de enfermagem desenvolverem também esta atividade. A administração dos medicamentos é de responsabilidade da equipe de enfermagem, que também monitora as possíveis reações adversas que podem surgir com a utilização do medicamento pelo paciente.

A partir desta percepção, observa-se que em todas as etapas envolvidas na utilização do medicamento pelo paciente, sempre estará envolvido algum profissional da área da saúde, que tem o compromisso e a responsabilidade de evitar erros de medicação por meio de atividades interdisciplinares, para o beneficio do paciente (OPITZ, 2006). 


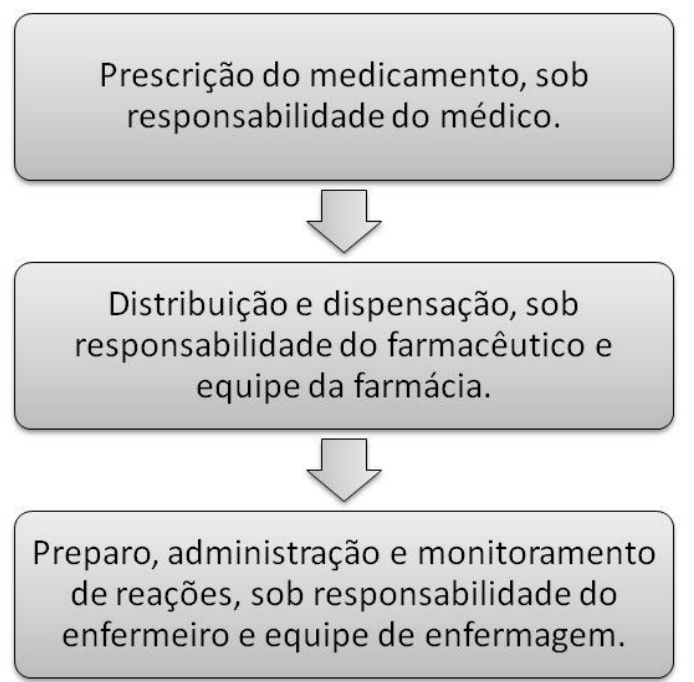

Figura 1. Processos e profissionais envolvidos no sistema de distribuição de medicamento. Fonte: Adaptado de OPITZ, 2006 .

Quando o medicamento não é administrado por algum motivo, ocorre outro processo denominado de estorno, que consiste na devolução dos medicamentos distribuídos às unidades para a farmácia hospitalar. As causas dessa devolução podem contemplar a não utilização do produto, uma vez que a prescrição pode prever que o uso somente será feito se necessário; a alta hospitalar; ou ainda, o óbito. O retorno do medicamento à farmácia se faz necessário para o controle de estoque efetivo e para a sua redistribuição, diminuindo custos do hospital. Porém, na prática o estorno pode apresentar uma série de irregularidades, que resultam da incorreta atribuição de medicamentos aos prontuários dos pacientes internados.

O número de erros evitáveis que ocorrem nos sistemas de saúde é cada vez mais evidente e inaceitável, e medidas precisam ser tomadas para mudar esse cenário (ROSA E PERINI, 2006). Estes erros podem ter por consequência a utilização irracional de medicamentos, contemplando prejuízos não apenas financeiros, mas também falhas na farmacoterapia.

Tendo em vista este contexto, o presente estudo foi conduzido em um hospital filantrópico de médio porte, em Santa Maria - RS, compreendendo a conferência do estorno dos medicamentos para a farmácia do hospital, tendo como objetivo analisar o sistema de medicamentos, buscando identificar causas e padrões de erros de devolução.

\section{2 - METODOLOGIA DA PESQUISA}

A pesquisa foi desenvolvida a partir de uma análise descritiva longitudinal. A descritiva teve por objetivo descrever as características de uma determinada população ou fenômeno ou também, estabelecer relações entre as variáveis, em um determinado momento do estudo (GIL, 2009).

O presente estudo foi realizado no período de 31 de Março a 25 de Novembro de 2011, em um hospital filantrópico da cidade de Santa Maria- RS. Foram verificadas as prescrições médicas de quatro unidades existentes no hospital, sendo os dados transcritos pra uma planilha, registrando os erros contidos nas devoluções dos medicamentos e materiais médicos. Esta pesquisa foi aprovada pelo CEP Unifra sob número 009/2010.2. 


\section{3 - RESULTADOS}

O sistema de medicação do Hospital em estudo segue várias etapas, conforme fluxograma descrito na figura 2.

Prescrição do medicamento, preenchendo itens como dose, via, frequencia, data e observações, em duas vias, realizada pelo médico.

Conferência da prescrição, aprazamento de horários, realizado pela enfermeira da unidade.

Entrega da prescrição à equipe da farmácia, conferência pelo farmacêutico, técnico de farmácia ou enfermagem.

Separação dos medicamentos que serão utilizados em 24 horas, acondicionamento destes no saco(embalagem) individual de cada paciente, anotação na segunda via da prescrição da baixa do medicamento para posterior digitação no estoque informatizado da farmácia.

Acondicionamento dos medicamentos e da primeira via da prescrição em caixas identificadas por unidade para que técnicos de enfermagem realizem a conferência do material e o transporte do mesmo até a unidade correspondente.

Recebimento dos medicamentos na unidade de enfermagem e administração nos pacientes, realizada pelos técnicos de enfermagem ou enfermeira responsável pela unidade.

Medicações não utilizadas em 24 horas são devolvidas ao saco do paciente e estornadas a farmácia.

Figura 2. Etapas e profissionais envolvidos no sistema de medicamentos do Hospital.

O hospital é constituído por 5 unidades de internação: unidade psiquiátrica (Unidade 100), unidade clínica adulta (Unidade 200), maternidade (Unidade 300), cirúrgica (Unidade 400) e unidade pediátrica (Unidade 500), sendo esta última não incluída na avaliação por seguir um modelo diferenciado de estorno de medicamentos. Durante a pesquisa, foram avaliadas 472 prescrições médicas, sendo que destas, 155 apresentaram erros nas devoluções de medicamentos e produtos médico hospitalares $(32,8 \%)$. Na Figura 3, observa-se o total de prescrições avaliadas por unidade e o número de erros apresentados em cada uma delas. 


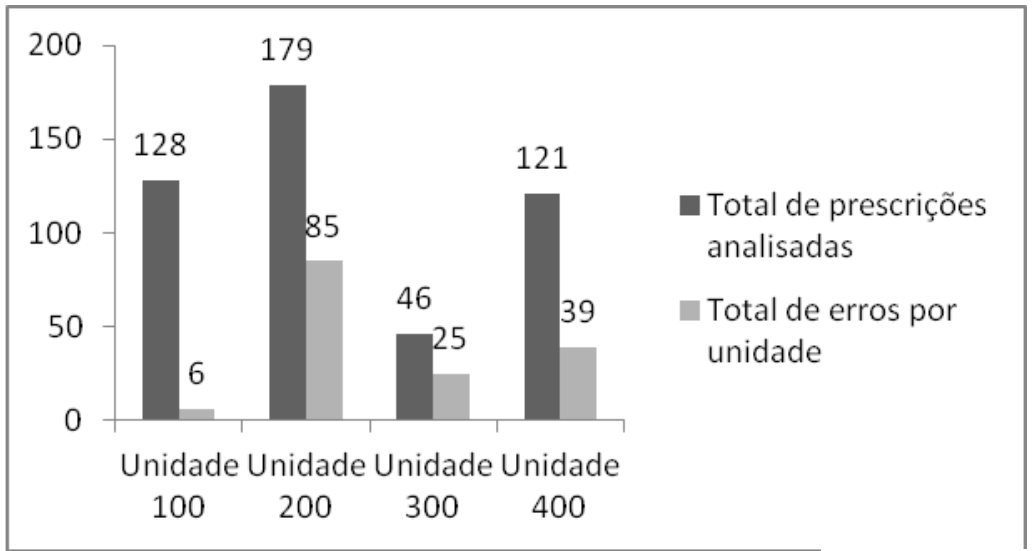

Figura 3. Total de prescrições avaliadas por unidade e respectivos erros em Santa Maria, RS (2011).

Acredita-se que a unidade 100 apresentou o menor porcentagem de erros, tanto em números absolutos quanto relativos, conforme as Figuras 3 e 4 , por ser a única unidade a possuir prescrição eletrônica, diminuindo a chance de interpretação errônea das mesmas. Além disso, a unidade faz uso, em sua maioria, de medicamentos controlados por internar pacientes com dependência química, o que torna a dispensação e o controle de aprazamento mais criteriosos e consequentemente, mais adequada em relação à devoluções de materiais não utilizados.

Quando comparados em relação à unidade de origem, em termos percentuais, pode-se visualizar grande quantidade de erros referente as unidades 400 e $200(54,35 \%$ e $47,49 \%$, respectivamente). Na unidade 400 , que compete à pacientes pós-cirúrgicos é possível inferir o alto número de devoluções pelas características dos medicamentos utilizados, em grande parte injetáveis. Graças a isto, as devoluções são em sua maioria referentes à materiais médico-hospitalares. Nestes casos, a padronização é a melhor medida para controlar o erro. Durante a coleta de dados, não havia padronização para diluição de medicamentos injetáveis. Tanto a padronizaçao quanto a atribuição de responsabilidades contribuem para a minimização do erro. Uma medida eficaz consiste na preparação e diluição de medicamentos injetáveis pela farmácia, pois simplifica o processo de administração e reduz as chances de erro de cálculo e de preparação, já que o preparo destas soluções é feito geralmente pela equipe de enfermagem em um ambiente agitado, repleto de distrações e não destinadas para este propósito. Além disso, o ambiente da farmácia seria mais facilmente adequado em relação à questões de segurança e esterilidade bem como conta com sistemas de apoio para garantir a exatidão na elaboração de cálculos (FEDERICO, 2004).

Pacientes internados na unidade 200 são em sua maioria adultos idosos, com quadros clínicos de elevado grau de complexidade, que resultam em prescrições extensas, muitas vezes incluindo mais de 10 medicamentos. A polimedicação, que é habitualmente reconhecida pelo uso concomitante de vários medicamentos, constitui um fator preditor positivo em relação ao tempo de internação, reinternação e mortalidade (GALVÃ O, 2006), além de aumentar as chances de erros de administração, que por sua vez se refletem em erros de devolução de medicamentos. Nestes casos, o erro na medicação ocorre em virtude da deficiência ou carência de conhecimento, desempenho abaixo do padrão, lapsos de memória ou deficiências no sistema, relacionados à profissionais, produtos, procedimentos, prescrições, uso e monitoramento de medicamentos (RODRIGUES, 2004). 


\section{Erros por Unidade (\%)}

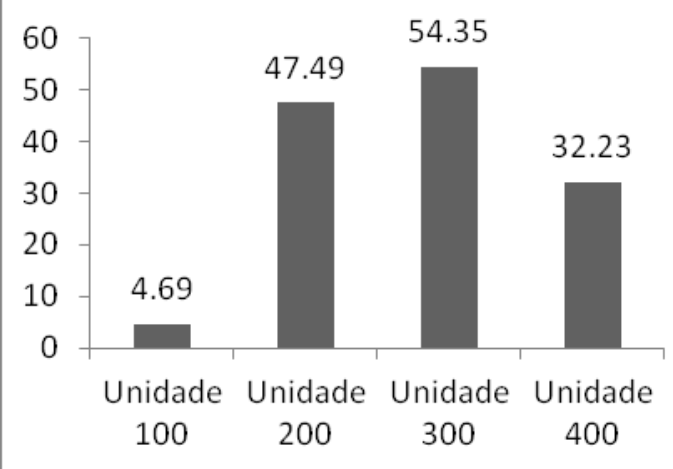

Erros por Unidade

(\%)

Figura 4. Porcentagem em erros apresentada por cada unidade em Santa Maria, RS (2011).

Durante o processo de coleta de dados, outras unidades além da unidade 100 passaram a emitir prescrições eletrônicas, ainda que parcialmente. Para melhoria do processo seria adequado adaptar ao sistema o uso do código de barras nas embalagens dos medicamentos, além da prescrição médica eletrônica, que constituem ferramentas importantes para a diminuir erros de medicação. A prescrição eletrônica elimina a dificuldade na leitura e no entendimento ocasionados pela letra ilegível do médico e possibilita que os erros de digitação sejam corrigidos no momento da elaboração da prescrição sem que, para isto, haja rasuras ou rabiscos que dificultam ainda mais o entendimento das informações (CASSIANI, FREIRE E GIMENES, 2003).

Apesar de ser uma ferramenta importante, a prescrição eletrônica não evita erros cometidos com prescrições que dão margem à dúvidas nos profissionais de enfermagem, ou naquelas em que há omissão de informações importantes como dose de apresentação e dose prescrita, horário, via de administração tempo de tratamento. Nestes casos, a educação e a capacitação permanente de médicos, enfermeiros e farmacêuticos, ou seja, do corpo clínico envolvido, permitirá a existência de prescrições mais completas e padronizadas, significando um grande avanço nas estratégias para redução de erros.

Conforme já mencionado, os erros mais comuns observados foram referentes às devoluções de produtos médico hospitalares. Acredita-se que esse maior número de erros ocorra devido a falta de uma padronização do uso de materiais para diluição e administração dos medicamentos. Outro aspecto analisado foi a falta de atenção nas devoluções realizadas pela equipe de enfermagem, que devolviam materiais diferentes dos dispensados à determinados pacientes, indicando que estes pertenciam a outros indivíduos.

Outro tipo de erro observado ocorreu devido ao aprazamento incorreto das precrições médicas, pois muitas vezes o medicamento era administrado ao paciente, porém não ocorria o registro na prescrição; desta forma, o medicamento que, segundo a prescrição, deveria ser devolvido à farmácia não se encontrava no caseiro do paciente, resultando em falha de devolução. Situação oposta ocorre quando o medicamento não é administrado e não retorna imediatamente ao caseiro do paciente, sendo devolvido ao caseiro de outro. Nesta situaçao, o número e o tipo de itens devolvidos era maior ou diferente do dispensado.

Além disso, outro fator determinante do número de erros de devolução diz respeito à periodicidade em que esta ocorre. É possivel que erros ocorram pela devolução atrasada de materias e medicamentos que ficam acumulados no posto da unidade de enfermagem, sujeito à evasão ou uso inadequado. Nestas condições, recomenda-se instituir como regra a devolução de medicamentos com maior brevidade possível, vinculada à requisição individual em nome de paciente, tendo identificados os responsáveis pela devolução. 
O desvio de medicamentos de unidades hospitalares foi estudado por Fidalgo e Silveira (2008), que avaliaram o uso indevido de medicamentos, especialmente psicotrópicos, por médicos. A pesquisa evidenciou que os médicos são mais suscetíveis ao uso abusivo de psicotrópicos quando comparados à população geral, sendo que $67,5 \%$ dos entrevistados declararam conhecer algum colega com problemas relacionados ao uso de substâncias, e 68,6\% dos clínicos julgam ser fácil o desvio de psicotrópicos da unidade de internação.

A utilização inadequada é essencialmente perigosa quando se trata de medicamentos de alto risco, como insulina, lidocaína, heparina, cloreto de potássio, agentes quimioterápicos, injeção de dextrose, narcóticos, agentes adrenérgicos, teofilina, imunoglobulina e bloqueadores neuromusculares. Nestes casos, a adoção de protocolos de rotulagem, armazenamento e administração são fundamentais para minimizar o risco de reações adversas que podem culminar com o óbito do paciente (FEDERICO, 2004).

Ao avaliarmos as devoluções em função do tempo de observação, pode-se perceber um aumento significativo das devoluções com erro na unidade 200, significativamente superior às demais unidades, porém, existe uma grande variação em cada unidade, em meses diferentes (Figura 5).

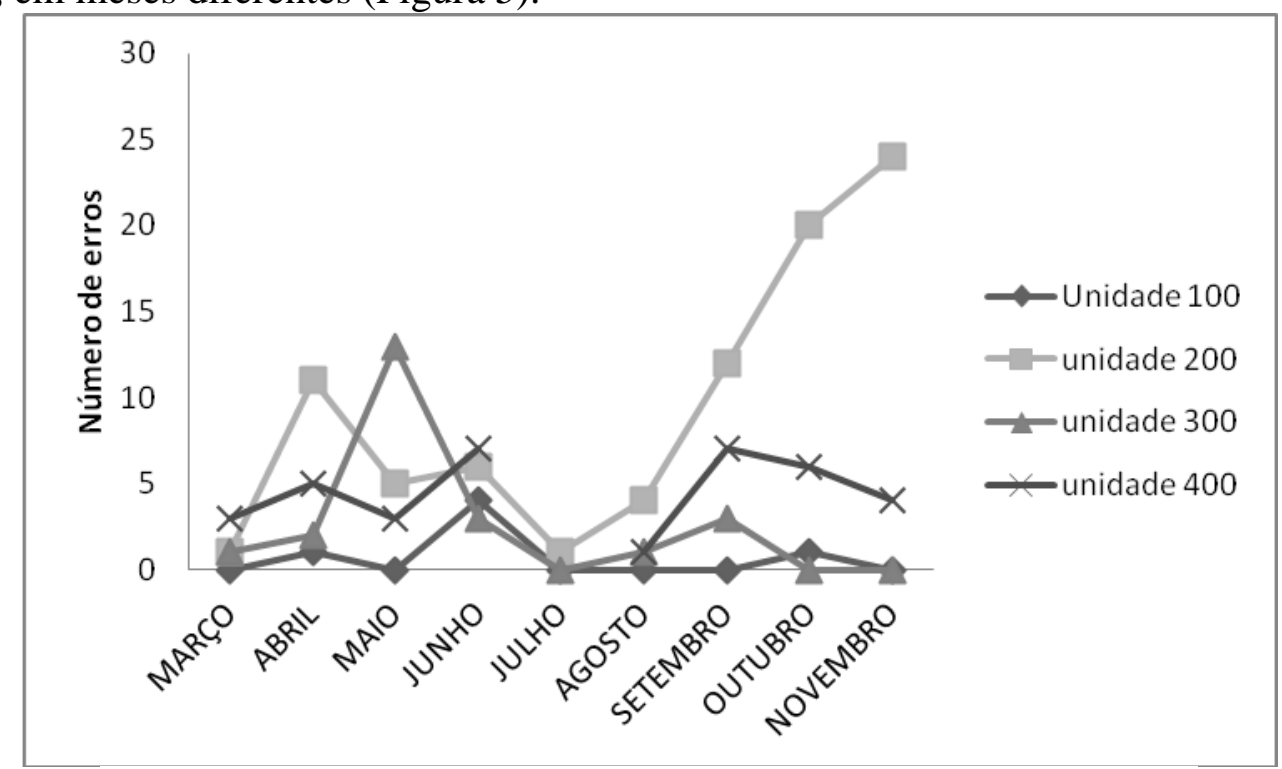

Figura 5. Erros apresentados em função do tempo, em Santa Maria, RS (2011).

Este resultado pode estar relacionado à mudanças no quadro de funcionários, não acompanhada de capacitação e padronização de atividades. Esta é uma necessidade proeminente, uma vez que a educação permanente deve agregar o pensar e o fazer dos trabalhadores com a finalidade de propiciar o crescimento pessoal e profissional dos mesmos e contribuir para a organização do processo de trabalho, através de etapas que possam problematizar a realidade e produzir mudanças, especialmente para a redução de erros que possam atingir o paciente (RICALDONI, 2006).

\section{5 - CONSIDERAÇÕES FINAIS}

De uma maneira geral, as farmácias hospitalares precisam trabalhar com uma grande quantidade de medicamentos, cujo fluxo compromete o planejamento e a gestão de estoques bem como a segurança do paciente. Com o objetivo de reduzir as oportunidades de erros de medicação, se faz necessário a adoção de algumas recomendações, dentre elas a adoção de procedimentos operacionais padrão, a adoção de dose unitária, a instituição de sistemas de preparação de soluções endovenosas na farmácia, desenvolver procedimentos especiais para fármacos de alto risco e de alto custo, promover a capacitação permanente e a educação 
farmacêutica. Além disso, a incorporação de tecnologias como a prescrição eletrônica e a utilização de leituras por máquinas (código de barras) no processo de administração de medicamentos também contribui para a minimização do estorno e consequente melhoria do fluxo de materiais.

A observação destas medidas, outrossim, só terá resultados favoráveis por meio da constante monitoração avaliação do fluxo dos materiais e que tem origem na farmácia do hospital, levando a diminuição de custos, além de aumentar a segurança do paciente usando a medicação e contribuir para o sucesso da farmacoterapia.

\section{6 - REFERÊNCIAS}

AGAPITO, N. Gerenciamento de Estoques em Farmácia Hospitalar. Anais: GELOG-UFSC, n.10, p.1-12, 2005.

CASSIANI, S.H.D.B; FREIRE, C.C.; GIMENES, F.R.E. A prescrição médica eletrônica em um hospital universitário: falhas de redação e opiniões de usuários. Rev Esc Enferm USP. v.37, n.4, p.1-60, 2003.

FEDERICO, F. Recomendações para as melhores práticas da medicalização: coalizão para prevenção de erros médicos de Massachusetts - EUA. In: Cassiani, SHB. A segurança dos pacientes utilizando medicação. São Paulo: Artes Médicas, 2004.

FIDALGO, T.M.; SILVEIRA, D.X. Uso indevido de drogas entre médicos: problema ainda negligenciado. J. Bras. Psiquiatr, v.57, n.4, p. 267-269, 2008.

GALVÃO, C. O idoso polimedicado - estratégias para melhorar a prescrição. Rev Port Clin Geral, v.22, p.747-52, 2006.

GIL, A.C. Como elaborar projeto de pesquisa. 4. ed. São Paulo: Atlas, 2009.

GONÇALVES, A.A.; NOVAES, M.L.O.; SIMONETTI, V.M.M. Otimização de farmácias hospitalares: eficácia da utilização de indicadores para gestão de estoques. Anais: ENEGEP, n.26, p.1-9, 2006.

MIASSO, A.I.; SILVA, A.E.B.C.; CASSIANI, S.H.B.; GROU, C.R.; OLIVEIRA, R.C.; FAKIH, F.T. O processo de preparo e administração de medicamentos: identificação de problemas para propor melhorias e prevenir erros de medicação. Revista Latino-americana de Enfermagem, v.14, n3, p.354-63, 2006.

OLIVEIRA, R.B.; MELO, E.C.P. O sistema de medicação em um hospital especializado no município do Rio de Janeiro. Esc. Anna Nery, v.15, n.3, p. 480-489, 2011.

OPITZ, S.P. Sistema de medicação: análise dos erros nos processos de preparo e administração de medicamentos em um hospital de ensino, 187f. Tese (Doutorado em Enfermagem Fundamental) - Programa de Pós-Graduação em Enfermagem Fundamental, Universidade de São Paulo, Ribeirão Preto, 2006.

RICALDONI, C.A.C; SENA, R.R. Educação permanente: uma ferramenta para pensar e agir no trabalho de enfermagem. Rev. Latino-Am. Enfermagem, v.14, n.6, 2006. 
RODRIGUES, M.L. Prescrição eletrônica de medicamentos. In: CASSIANI, S.H.B. A segurança dos pacientes utilizando medicação. São Paulo: Artes Médicas, 2004

ROSA, M.B.; PERINI, E. Erros de medicação: Quem foi? Revista Associação Medica Brasileira, v.49, n.3, p. 335-341,2003. 